\title{
HF spectrum occupancy and antennas
}

\author{
António Casimiro ( ${ }^{(1)}$, Joaquim Azevedo $\left({ }^{2}\right)$, Lefteris Economou $\left({ }^{3}\right)$, \\ Haris Haralambous $\left({ }^{4}\right)$, Ersin Tulunay $\left({ }^{5}\right)\left({ }^{6}\right)$, Yurdanur Tulunay $\left({ }^{5}\right)$, Yildirim Bahadirlar $\left({ }^{6}\right)$, \\ A. Serdar Türk $\left(^{6}\right)$ and E. Michael Warrington $\left(^{7}\right)$ \\ (') CEFAT, DEEI, FCT, University of Algarve, Portugal \\ $\left.{ }^{2}\right)$ Engineering and Mathematics Department, University of Madeira, Portugal \\ (3) Intercollege, Cyprus \\ $\left(^{4}\right)$ Frederick University, Cyprus \\ $\left(^{5}\right)$ Middle East Technical University, Ankara, Turkey \\ $\left.{ }^{6}{ }^{6}\right)$ TÜBITTAK, MAM, Kocaeli, Turkey \\ (') Department of Engineering, University of Leicester, Leicester, UK
}

\begin{abstract}
This paper deals with the research made during the COST 296 action in the WG2, WP 2.3 in the antennas and $\mathrm{HF}$ spectrum management fields, focusing the Mitigation of Ionospheric Effects on Radio Systems as the subject of this COST action.
\end{abstract}

Key words Antenna Analysis - Antenna Synthesis Fourier Relation - Spectrum Management

\section{Antennas}

\subsection{Introduction}

The «Small Translation Theorem» developed by Prof. Francisco Grilo in his classes at the University of Porto gave rise to a new procedure of dealing with antennas field that gives a new insight into the field and opens easy procedures for practical applications. The Ph.D. Thesis of several professors (Casimiro, 1990; Azevedo, 2001; Xianfeng, 2004) covered the different aspects of this new procedure as well as other publications.

Since the propagation of signals through the ionosphere can be significantly affected by its

Mailing address: Prof. Doutor António Casimiro, CEFAT, DEEI, FCT, University of Algarve, Campus de Gambelas, 8000-117 Faro, Portugal; e.mail: acasimi@ualg.pt presence, antenna arrays play an important role in the mitigation of ionospheric effects. In many communication systems it is desirable for the antenna to have a narrow beam with very low side lobe levels. Other situations, such as GPS satellites, require shaped beam patterns. The interference could be mitigated by imposing appropriated nulls on the radiation pattern and controlling the sidelobe levels.

This paper summarizes the basic concepts and presents one application example covering the application of the new procedure to the antenna field, developed during the COST 296 action. The references cover the remaining developments.

The understanding of these situations give the possibility of new mitigation procedures not only in the COST 296 area, but also in ther areas, so opening an inter COST collaboration as was already being made with COST 284 and now with COST IC 0603.

Besides the one-dimensional antenna distributions, the Fourier Relation is also suited for the two-dimensional case, and for the general 3D distributions of sources. Even if all the situations are fully covered by the developed Fouri- 
er Relation, it is useful to work in the particular aspects of one, two and three dimension antenna distribution cases.

Going for the two-dimensional case, a planar array has two dimensions of control. Considering that the relationship between the array factor and the array excitations for the far field region is a Fourier transform in the appropriate variables (Casimiro and Azevedo, 2005), much of the work achieved has been to obtain appropriated synthesis techniques based on this relation.

In a previous study the array elements were in a rectangular grid (Azevedo, 2007). Considering the signal processing theory, for this case it is possible to apply the sampling theorem to the array factor.

Therefore, this kind of geometry permits the use of the Fast Fourier Transform algorithm to make the involved calculations. On the other hand, since a ring array offers advantages using fewer elements than a filled planar array and a geometry that facilitates 360 degrees scanning, (Hansen, 1998), the Fourier relation can be applied to planar arrays with the ele- ments in a circular grid. To deal with circular apertures the Hankel transform could be considered (Elliot, 1981).

\subsection{Basic Concepts}

When a distribution of point sources is given, a spatial translation of any source only modifies the produced field in the phase, according to the eq. 1.1 (Casimiro, 1990):

$$
\begin{aligned}
& \underline{c}_{1}(\mathbf{d}) \longrightarrow \underline{F}_{1}(\theta, \phi) \\
& \underline{a c} \underline{c}_{1}\left(\mathbf{d}+\mathbf{d}_{0}\right) \longrightarrow \underline{a} \underline{e}^{j \beta_{0 . \mathbf{d}}} \underline{F}_{1}(\theta, \phi)
\end{aligned}
$$

where $\beta_{\mathrm{O}}=2 \pi / \lambda, \lambda$ is the wavelength "." means the dot product. It can be said that all the source distribution is under the Small Translation Theorem application. When it occurs, the array pattern is always a window in the Inverse Fourier Transform of the source distribution if we use a convenient representation of the variables involved.

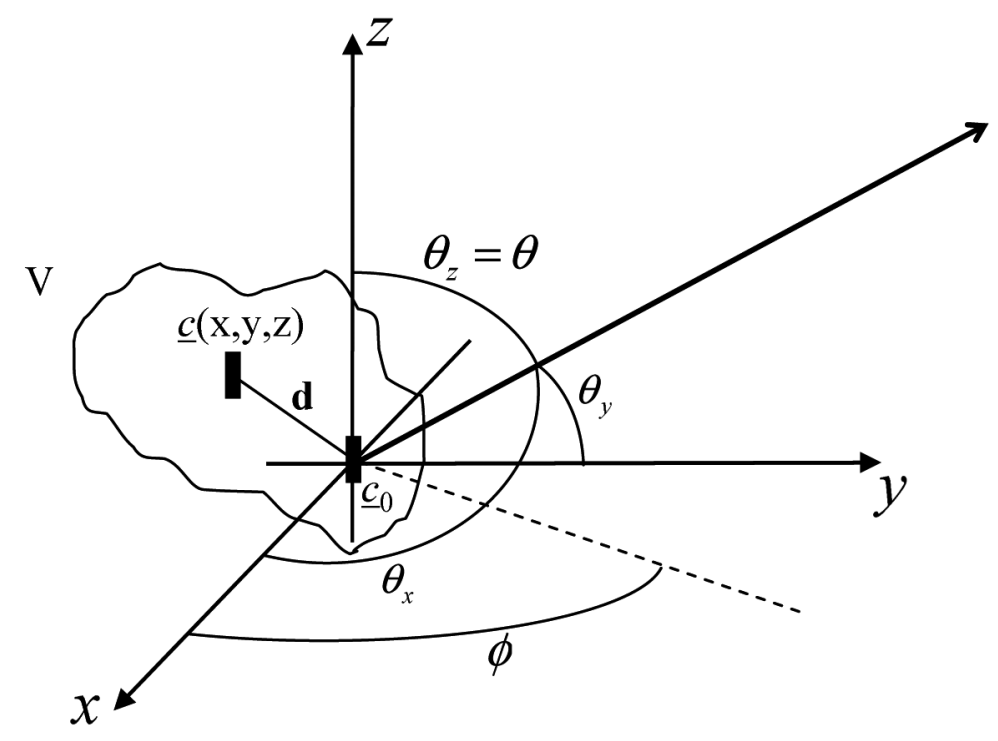

Fig. 1. The Fourier Relation: the 3D general situation. 
This was called the Fourier Relation (Grilo and Casimiro, 1991).

The general case of three-dimensional source distribution is represented in fig. 1 and the Fourier Relation is given by

$$
\begin{aligned}
& \underline{F}\left(\beta_{x}, \beta_{y}, \beta_{z}\right)=\int_{-\infty}^{\infty} \int_{-\infty}^{\infty} \int_{-\infty}^{\infty} \underline{c}(x, y, z) e^{j\left(\beta_{x} x+\beta_{y} y+\beta_{z} z\right.} d x d y d z \\
& =(2 \pi)^{3} F^{-1}[\underline{c}(x, y, z)] \\
& \underline{c}(x, y, z)=\int_{-\infty}^{\infty} \int_{-\infty}^{\infty} \int_{-\infty}^{\infty} \underline{F}\left(\beta_{x}, \beta_{y}, \beta_{z}\right) e^{-j\left(\beta_{x} x+\beta_{y} y+\beta_{z} z\right)} d \beta_{x} d \beta_{y} d \beta_{z} \\
& =\frac{1}{(2 \pi)^{3}} F\left[\underline{F}\left(\beta_{x}, \beta_{y}, \beta_{z}\right)\right]
\end{aligned}
$$

where $\underline{c}(x, y, z)$ is the source distribution, $\underline{F}\left(\beta_{x}, \beta_{y}, \beta_{z}\right)$ is the array factor, $F$ means the Fourier transform, the bar under the variable indicates the temporal Fourier transform and $\beta_{x}=\beta \cos \left(\theta_{x}\right), \beta_{y}=\beta \cos \left(\theta_{y}\right)$ and $\beta_{z}=\beta \cos \left(\theta_{z}\right)$.

The one-dimensional case and two-dimensional case are particular cases of this tridimensional one, as it is obvious. This approach has been successfully used for the research in the antennas and propagation fields, namely during this action time as can be seen from the references. Next it will be illustrated by applying it at a circular antenna array.

\subsection{Circular arrays of Antennas}

A direct application of the Fourier Relation expressed by eq. 1.2 is to planar arrays. In order to control the radiation pattern, Azevedo (2007) presented a technique that controls directly the peaks of a rectangular array factor. In the present work, the Fourier Relation is applied to circular arrays.

For two-dimensional systems that exhibits circular symmetry is more appropriate to deal with the Hankel transform. This transform, also called Fourier-Bessel transform, is defined by (Cohen, 1992).

$$
\begin{gathered}
F_{v}(s)=\int_{0}^{\infty} f(r) J_{v}(s r) r d r \\
f(r)=\int_{0}^{\infty} F_{v}(s) J_{v}(s r) s d s
\end{gathered}
$$

with $J_{v}(\alpha)$ the Bessel function of first kind and order $v$. The two-dimensional Fourier transform can be related with the Hankel transform (Wylie and Barret, 1995). If $F(u, v)$ is the Fourier transform of $f(x, y)$, and exists radial symmetry, it means $f(x, y)=f(r)$ is independent of the $\phi$ variable, with $r^{2}=x^{2}+y^{2}, x=r \cos (\phi), \mathrm{y}=r \sin (\phi)$, and if $F(u, v)=F(q)$, then

$$
\begin{aligned}
& F(q)=2 \pi \int_{0}^{\infty} f(r) J_{0}(q r) r d r \\
& f(r)=\frac{1}{2 \pi} \int_{0}^{\infty} F(q) J_{0}(q r) q d q
\end{aligned}
$$

These equations constitute the Hankel transform for Bessel functions of order $v=0$.

In the following, the Fourier Relation is analyzed for planar arrays with circular symmetry. Considering the polar coordinate system,

$$
\begin{array}{ll}
x=\rho \cos (\varphi) & \beta_{x}=\xi \cos (\psi) \\
y=\rho \cos (\varphi) & \beta_{y}=\xi \sin (\psi) \\
\rho^{2}=x^{2}+y^{2} & \xi^{2}=\beta_{x}^{2}+\beta_{y}^{2}
\end{array}
$$

for a continuous source distribution, $\underline{c}(\rho, \varphi)$, the array factor defined is:

$$
\begin{aligned}
& \underline{F}(\xi, \psi)=\int_{0}^{\infty} \int_{0}^{2 \pi} \underline{c}(\rho, \varphi) e^{j \xi \xi \cos (\psi) \cdot \rho \cos (\varphi)+\xi \sin (\psi) \cdot \rho \sin (\varphi)]} \rho d \rho d \varphi \\
& =\int_{0}^{\infty} \int_{0}^{2 \pi} \underline{c}(\rho, \varphi) e^{j \rho \xi \cos (\psi-\varphi)} \rho d \rho d \varphi
\end{aligned}
$$

If the source distribution only depends on the distance to the origin and considering that (Cohen, 1992)

$$
J_{0}(x)=\frac{1}{2 \pi} \int_{0}^{2 \pi} e^{j x \cos (\alpha)} d \alpha
$$

then

$$
\begin{aligned}
& \underline{F}(\xi)=\int_{0}^{\infty} \underline{c}(\rho)\left[\int_{0}^{2 \pi} e^{j \rho \xi \cos (\psi-\varphi)} d \varphi\right] \rho d \rho \\
& =2 \pi \int_{0}^{\infty} \underline{c}(\rho) J_{0}(\rho \xi) \rho d \rho
\end{aligned}
$$

This is the well-known result for a continuous disc source distribution, being similar to the one given by eq. 1.4. As noted by several authors (Elliot, 1981), since the source distribution does not depend on $\varphi$, the array factor is also independent of $\psi$. 
For a discrete planar array with $M$ concentric rings, as represented in fig. 2, using the changing of variables defined in eq. 1.5

$$
\begin{aligned}
& \underline{F}(\xi, \psi)=\sum_{m=0}^{M-1} \sum_{n=0}^{N_{m}-1} c\left(\rho_{m}, \varphi_{n}\right) e^{j\left[\xi \cos (\psi), \rho_{m} \cos \left(\varphi_{n}\right)+\xi \sin (\psi) \cdot \rho_{m} \sin \left(\varphi_{n}\right)\right]} \\
& =\sum_{m=0}^{M-1} \sum_{n=0}^{N_{m}-1} c\left(\rho_{m}, \varphi_{n}\right) e^{j m_{m} \xi \cos \left(\psi-\varphi_{n}\right)}
\end{aligned}
$$

with $\varphi_{n}=2 \pi n / N_{m}$ and $N_{m}$ is the number of elements in ring $m$.

As performed for rectangular grids, the previous result could also be obtained sampling the continuous definition with Diracs impulses in the positions of the array elements divided by the ring radius. Considering the decomposition in Fourier series,

$$
e^{j \rho_{m} \xi \cos \left(\psi-\varphi_{n}\right)}=\sum_{k=-\infty}^{\infty} j^{k} J_{k}\left(\rho_{m} \xi\right) e^{j k\left(\psi-\varphi_{n}\right)}
$$

for an array distribution that only varies with $\rho$, eq. 1.9 becomes

$$
\begin{aligned}
& \underline{F}(\xi)=\sum_{m=0}^{M-1} \sum_{n=0}^{N_{m}-1} \underline{c}\left(\rho_{m}, \varphi_{n}\right) \sum_{k=-\infty}^{\infty} j^{k} J_{k}\left(\rho_{m} \xi\right) e^{j k\left(\psi \frac{2 \pi}{N_{m}} n\right)} \\
& =\sum_{m=0}^{M-1} \underline{c}\left(\rho_{m}\right) \sum_{k=-\infty}^{\infty} j^{k} J_{k}\left(\rho_{m} \xi\right) e^{j k \psi} \sum_{n=0}^{N_{m}-1} e^{-j \frac{2 \pi}{N_{m}} k n}
\end{aligned}
$$

Since the summation in $n$ is equal to $N_{m}$ if $k=N_{m} p, p=0, \pm 1, \pm 2, \ldots$, and zero otherwise, the array factor is given by

$$
\underline{F}(\xi, \psi)=\sum_{m=0}^{M-1} N_{m} \underline{c}\left(\rho_{m}\right) \sum_{p=-\infty}^{\infty} J_{N_{m p}}\left(\rho_{m} \xi\right) e^{j N_{m p}\left(\psi+\frac{\pi}{2}\right)}
$$

Although the series in $p$ is infinite, usually the first few terms are enough to produce a good approximation for the array factor. If only the Bessel function of zero order is considered for eq. 1.12, the result is similar to sampling (1.8), corresponding to the array factor of a continuous distribution. The contribution of higher order terms diminishes with increasing $N_{m}$. On the other hand, in practical situations the number of elements in each circle must be enough to produce a good compromise between the beam width and the sidelobe levels. In fact, for a rectangular grid the distance between elements is usually around $\lambda / 2$ to $\lambda$ to avoid grating lobes inside the visible window. In the same way for circular arrays, increasing the distance between elements introduces sidelobes with high values inside the visible window for radiation. Figure 3

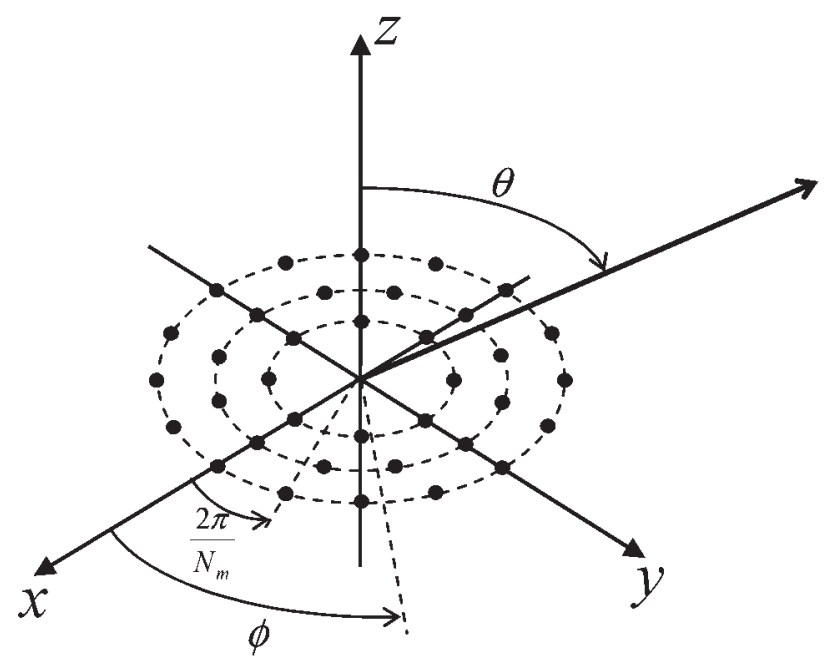

Fig. 2. Concentric rings planar array. 
a)

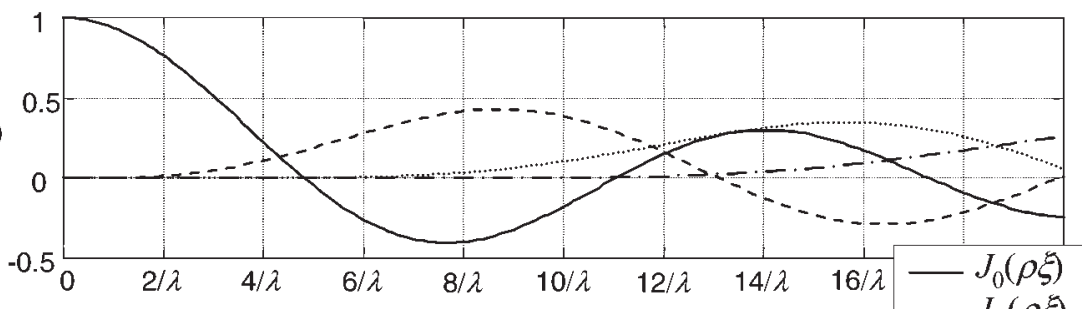

b)

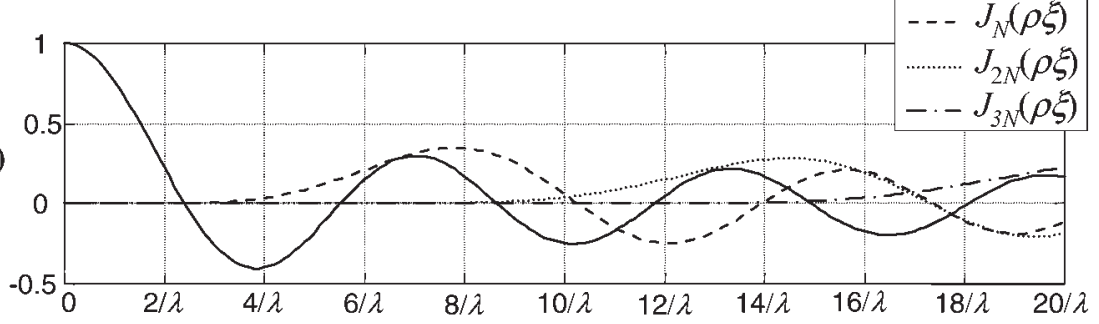

c)

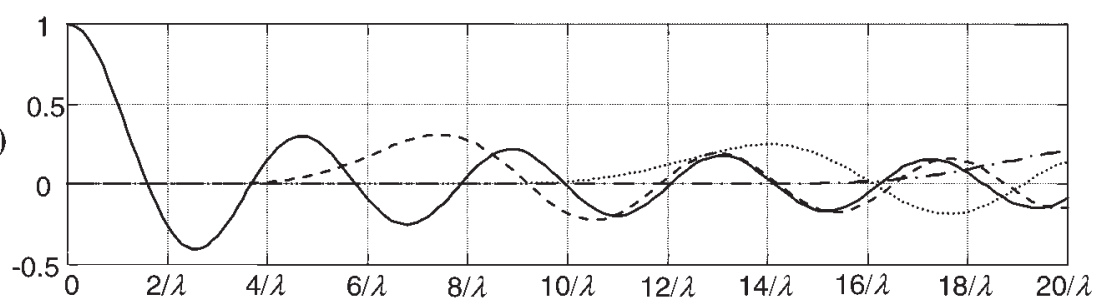

Fig. 3. First terms of the array factor infinite series: a) $r=0.5 l$; b) $r=l$; c) $r=1.5 l$.

shows the contribution of each Bessel function term represented in eq. 1.12 as a function of $\xi$ for a distance between elements around a wavelength. Three ring radiuses were considered. Since the distance between elements can be approximated by the portion of the arc around the ring, for a specified distance between elements around $\lambda / 2$ the number of elements is given by $N_{m}=4 \pi \rho_{m} / \lambda$. Since the visible window region is equal or less than $\beta=2 \pi / \lambda$, for this situation the array factor is given practically by the first terms of the summation.

The source distribution of the discrete array can be calculated using (eq. 1.2). Considering the changing of variables defined in (eq. 1.5), the following is obtained

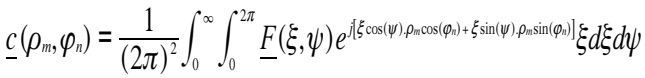

$$
\begin{aligned}
& =\frac{1}{(2 \pi)^{2}} \int_{0}^{\infty} \int_{0}^{2 \pi} F(\xi, \psi) e^{j \operatorname{jon} \xi \cos (\psi-\varphi)} \xi d \xi d \psi
\end{aligned}
$$

In contrast to the continuous case, as it can be verified from (eq. 1.12), the array factor of a discrete planar has both dependence of $x$ and $y$. Only when the approximation to the Bessel function of order zero is accepted there is independence of $y$ and the source distribution is given by

$$
\begin{aligned}
& \underline{c}\left(\rho_{m}\right)=\frac{1}{(2 \pi)^{2}} \int_{0}^{\infty} \underline{F}(\xi)\left[\int_{0}^{2 \pi} e^{j \rho_{m} \xi \cos \left(\psi-\varphi_{m}\right)} d \psi\right] \xi d \xi \\
& =\frac{1}{2 \pi} \int_{0}^{\infty} \underline{F}(\xi) J_{0}\left(\rho_{m} \xi\right) \xi d \xi
\end{aligned}
$$

In this case, the source distribution can be obtained using the Hankel transform of zero order. 


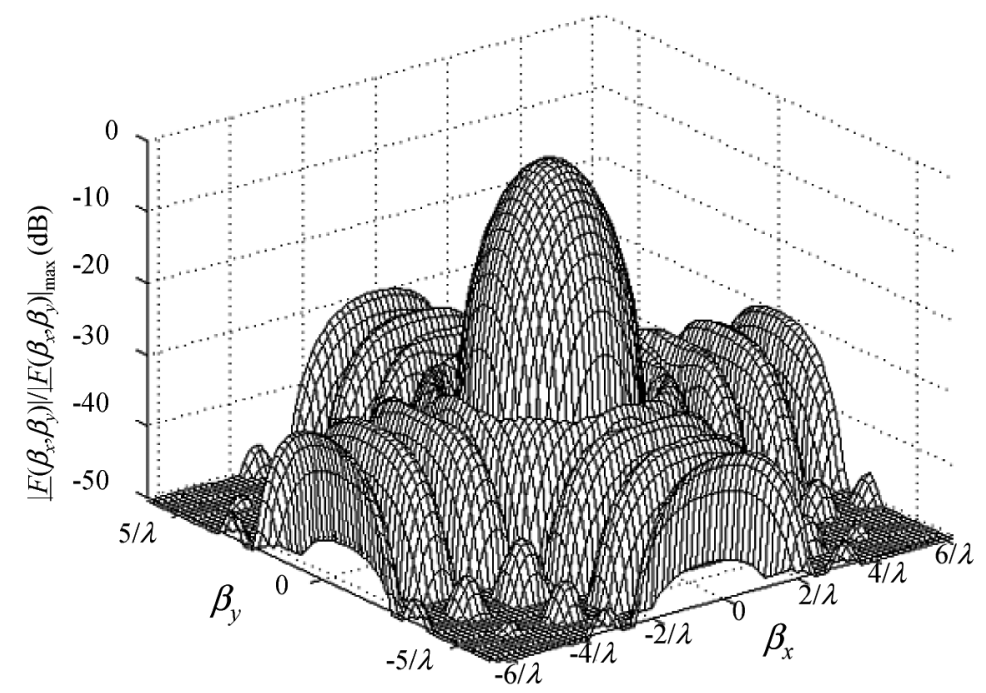

Fig. 4. Array factor for an array in a rectangular grid with sidelobe level of $-30 \mathrm{~dB}$.

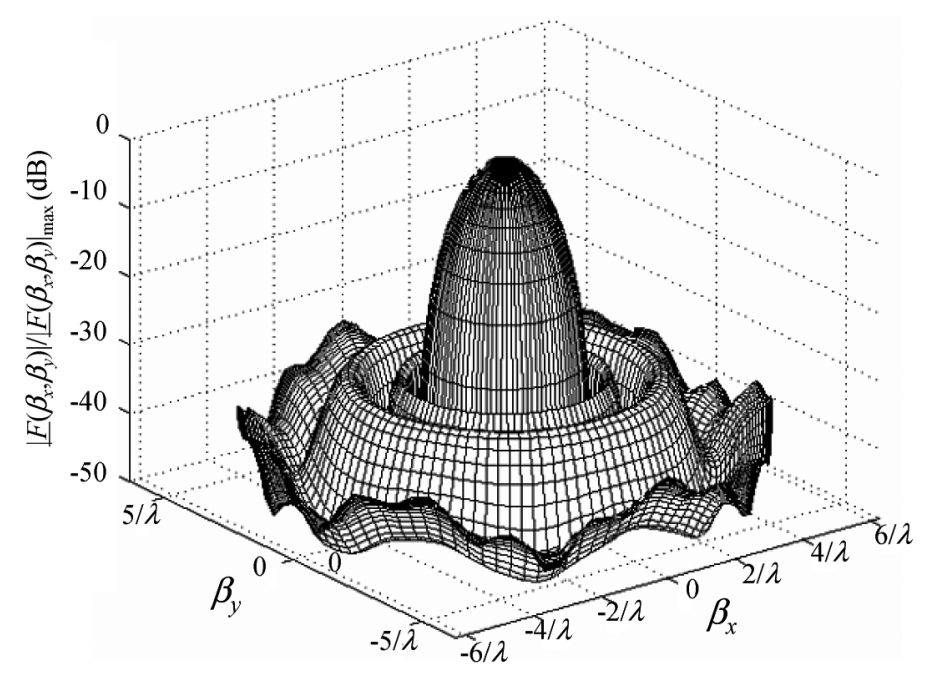

Fig. 5. Array factor for a concentric rings array.

\subsection{Results}

For antenna arrays, an array with 81 elements and distance between elements of $0.5 \lambda$ is considered. The method presented in (Azevedo, 2007) gives the result of fig. 4 , for sidelobe lev- els $30 \mathrm{~dB}$ below the main lobe.

For a planar array with circular symmetry $M=6$ concentric rings were considered and the same number of elements and positions as the previous example. One element is in the origin. The rings have $6,12,18,24$ and 20 elements, 


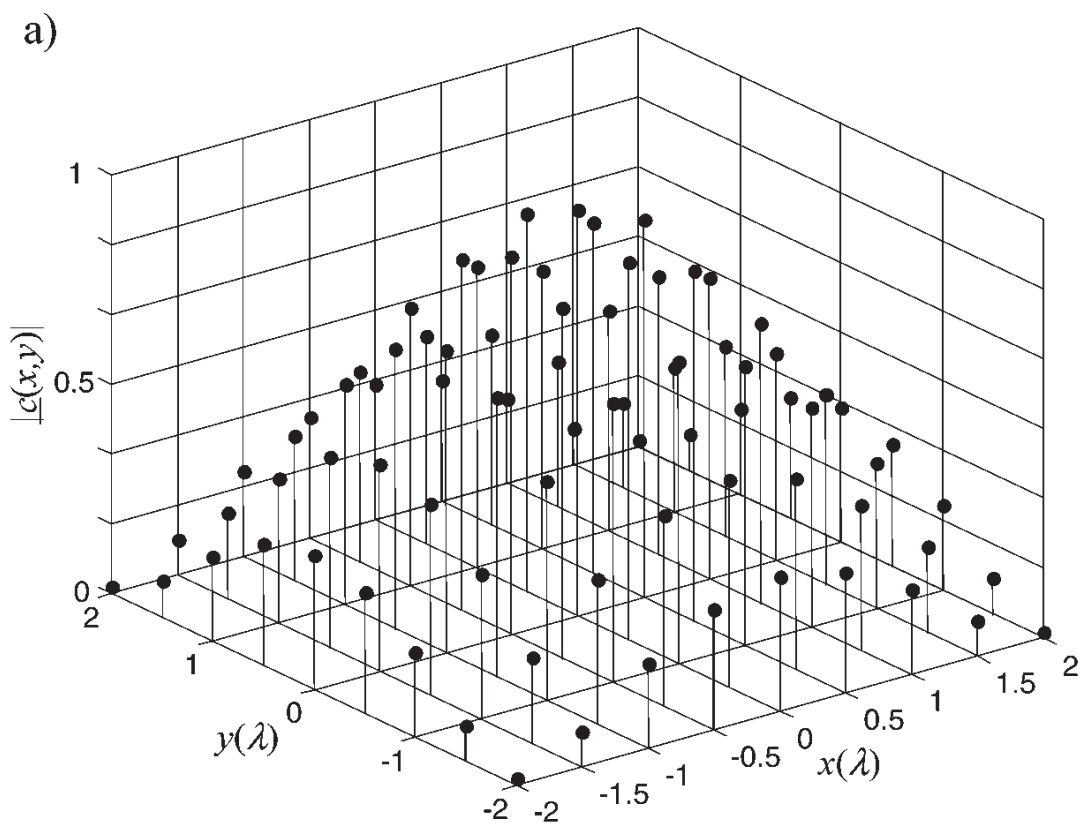

b)

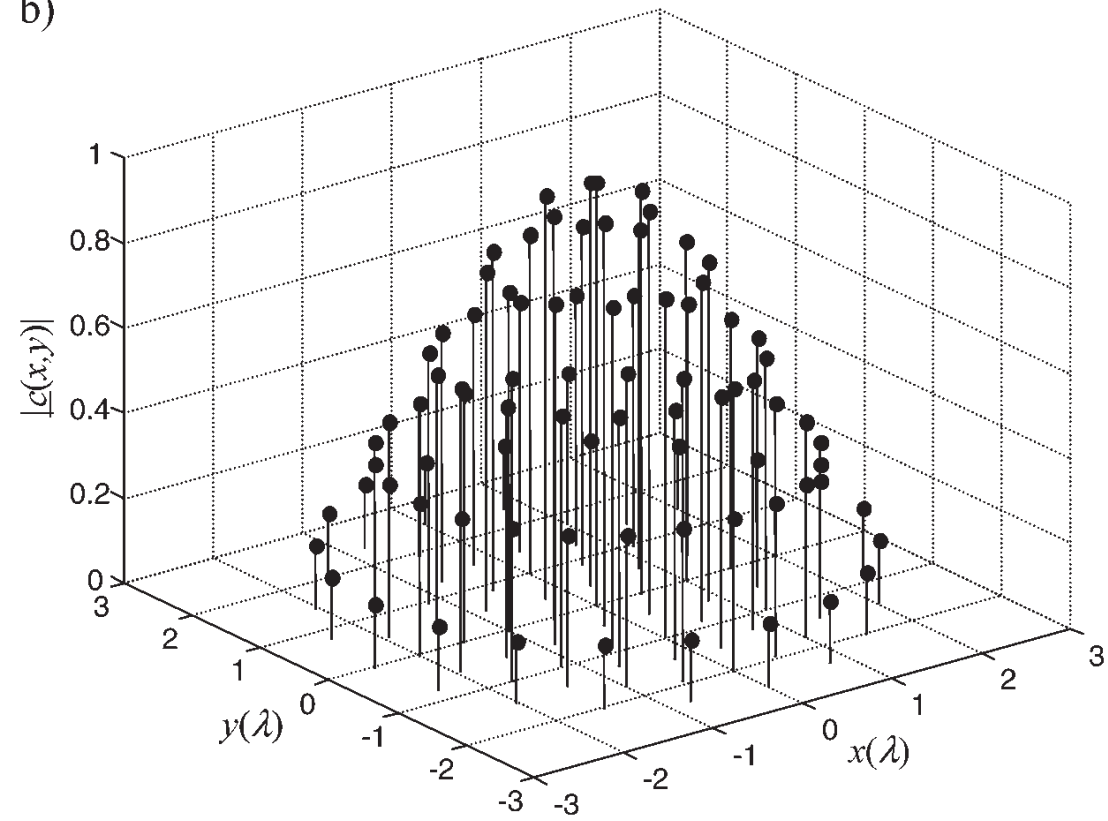

Fig. 6. Source distribution: a) rectangular array; b) circular array. 
respectively. The distance between rings is $0.5 \lambda$ and the distance between elements of the inner rings is around $0.52 \lambda$. For the last ring the distance between elements is $0.78 \lambda$. For comparison with the previous example, an array factor with a similar beam width was considered. Since the desired array factor does not depends on $\phi$ the source distribution was calculated using (eq. 1.14), considering that $\underline{F}(\xi)=1$ for $\xi<1.3 \lambda$ and $\underline{F}(\xi)=0$ otherwise. Figure 5 shows the array factor. Only two terms of (eq. 1.12) are enough to produce this result. Compared with the rectangular grid array, the higher sidelobe level for a uniform distribution is around $13 \mathrm{~dB}$ whilst for the concentric circular array the higher sidelobe level was around $-18 \mathrm{~dB}$. As it can be observed from fig. 8 , the sidelobe levels are $30 \mathrm{~dB}$ below the main lobe.

Therefore, based on the array factor configuration, in some applications the synthesis technique can explore its symmetry in a simpler manner.

The source distributions for both arrays are given in fig. 6 . The feed system of the circular array is simpler than the one of the rectangular grid since several elements have the same excitation.

\subsection{Conclusions}

During this COST 296 action time, the «Fourier Relation» was successfully applied to two dimensional antenna arrays to control the radiation pattern that is one of the main tools for Mitrigation of Ionospheric Effects on Radio Systems. The common field application makes the research suitable for collaboration with other COST actions as was done with COST 284 and COST IC 0603.

\section{Spectrum occupancy}

\subsection{Introduction}

A long-term joint UK-Swedish-German project has been undertaken on the measurement and analysis of HF spectral occupancy over northern Europe. Measurements that resulted from this project have been used to further investigate spectral occupancy in the frame of COST 296. In this COST action the research effort focused on two areas: a) procedures for statistically modelling spectral occupancy, with final stable-day and stable-night models for northern Europe, using calibrated monopole antennas (Economou et al., 2005) corresponding to weekly occupancy measurements; b) investigation into the possible application of artificial neural networks to predict spectral occupancy in the HF spectrum (1.6-30MHz). In particular, a study of the development of ITU allocation models for the diurnal variation of HF spectral occupancy over Sweden is made (Haralambous and Papadopoulos, 2007; Haralambous et al., 2008). Furthermore the application of Neural Networks as a means of optimising the reliability of HF groundwave communication systems by forecasting the detrimental effect of interference from other users is examined (Haralambous and Papadopoulos, 2008). This paper also presents atmospheric noise measurements over the HF band during the 29 March 2006 total solar eclipse in Antalya (Tulunay, E. et al., 2006b).

\subsection{Measurements during the 29 March 2006 total solar eclipse week in the eastern Mediterranean region}

Measurements over the HF band during the 29 March 2006 total solar eclipse in Antalya $\left(36^{\circ} \mathrm{N}, 30^{\circ} \mathrm{E}\right)$ Turkey were conducted from the channel occupancy and atmospheric noise points of view.

The whole HF band ranging from 1 to 30 $\mathrm{MHz}$ has been swept using $10 \mathrm{kHz}$ peak and $200 \mathrm{~Hz}$ average detectors of a certified EMI receiver (HP-8542E) equipped with a calibrated active monopole antenna (HE-011) (MCM4, 2006 (p. 31-35); Tulunay et al., 2006a; 2006b; Tulunay E., 2007).

Figure 7 shows the atmospheric noise variation almost at the time the total solar eclipse. The results indicate that during the total eclipse the noise level exhibited a different pattern. Qualitatively, «the eclipse» values somehow 


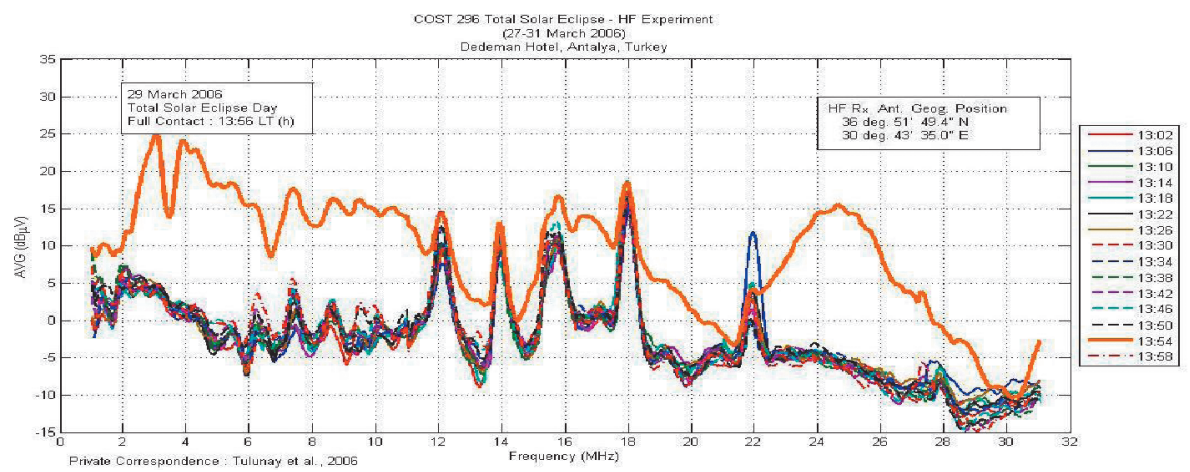

Fig. 7. The atmospheric noise level on the time of the total eclipse.
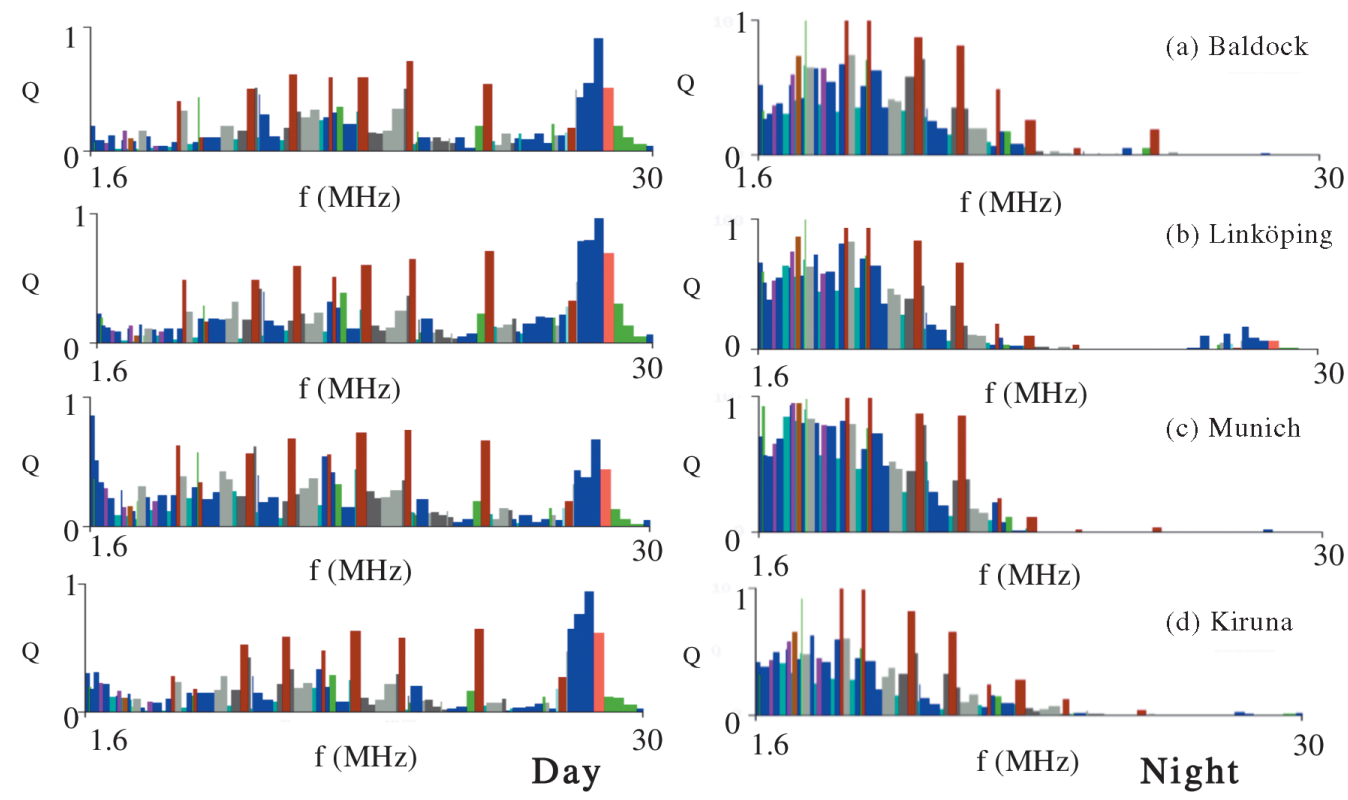

Fig. 8. Examples of measured day and night congestion at the $-117 \mathrm{dBm}$ signal threshold level.

represent the characteristic behavior of the night-time. After the total eclipse the atmospheric noise level returned back to its preeclipse pattern both in magnitude and configuration (MCM4, 2006 (p. 31-35).

\subsection{Measurement of HF spectral occupancy over Europe}

For the purpose of taking occupancy measurements the HF spectrum was divided into 95 allocations, which are shared by twelve different types of user. These allocations are based 
on the ITU frequency allocations for Region 1 ITU WARC (1979), but include some variation modifications as they were interpreted in accordance to the measurement of spectral occupancy. The measure of occupancy used is congestion, which is defined as the probability of placing at random, a bandpass filter of a given bandwidth in a given ITU defined frequency allocation, such that the RMS value of the filter output signal exceeds a predefined threshold level.

\subsubsection{Measurement system}

The rural measurement sites were situated at Baldock (UK), Linköping and Kiruna (Sweden) and Munich (Germany). The measurement receiver was the Rohde and Schwarz R\&S ESH3, and the calibrated active measurement antenna at each site was a low-angle monopole (R\&S HE010). The system at each site, and the

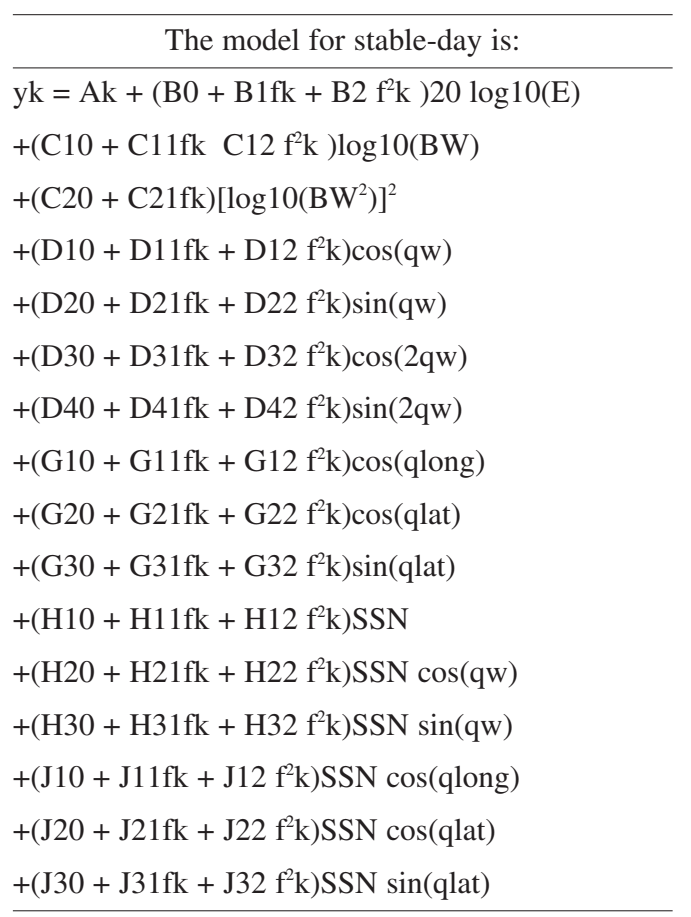

measurement procedure have been described previously Gott, G.F. et al. (1997). At each site, during a weekly congestion measurement, the receiver frequency was incremented through each frequency allocation, using typically a $1 \mathrm{kHz}$ step, a $1 \mathrm{kHz}$ measurement bandwidth and a $1 \mathrm{~s}$ dwell time. The fraction of the allocation spectral width for which the RMS signal level at each step exceeds a defined fieldstrength threshold level defines the occupancy or congestion for that frequency allocation, for the corresponding threshold level, measurement bandwidth, time of day, and sunspot number. Figure 8 shows examples of HF spectral occupancy at all sites where different types of user appear in different colour.

Diurnal measurements of occupancy were obtained once a week provided that no other measurement campaigns were in progress. using a $90 \mathrm{~ms}$ dwell time at each increment. The HF spectrum was monitored in less than an hour by employing a variable frequency increment algorithm.

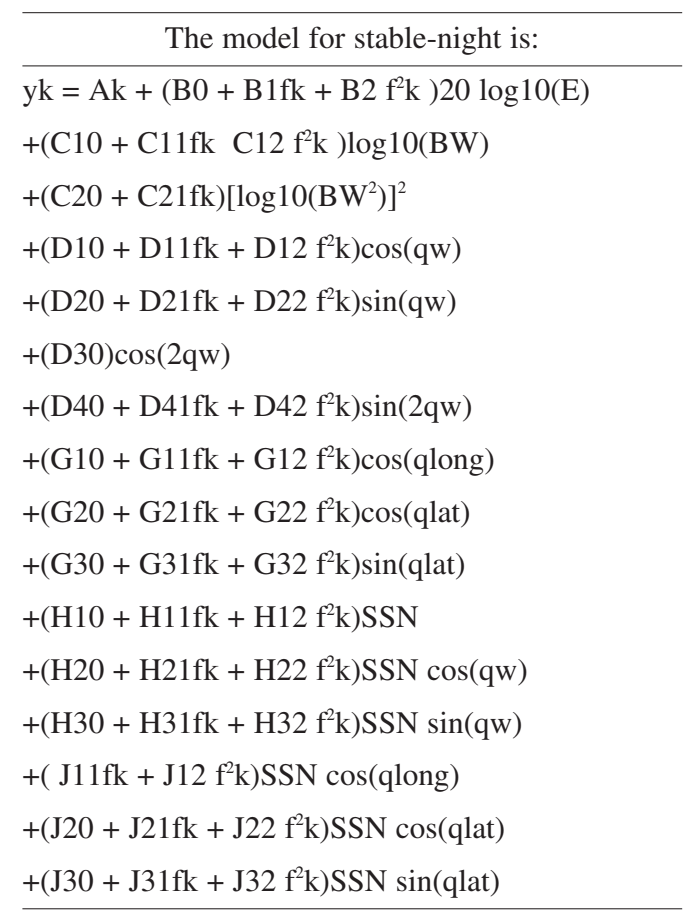


This compromise does not affect significantly the resulting congestion values, based on RMS signal measurements. Figure 9 shows a diurnal congestion measurement for allocations residing in the upper and lower regions of the HF spectrum.

\subsection{Models of HF spectral occupancy over a sunspot cycle}

The statistical occupancy models are based on weekly measurements made since 1990, which apply to stable-day and stable-night ionospheric conditions when using a calibrated monopole antenna. Congestion measurements for every frequency allocation were made at each site, typically every 14 days, for both stable-day and stable-night ionospheric conditions.

These conditions corresponded to two periods, each of duration about three hours, centered in time on local midday and on local midnight, and give rise to very different occupancy characteristics.

The models may be used in conjunction with frequency predictions to advise operators on the occupancy they may encounter, and how this varies with field-strength threshold level, frequency, time, bandwidth, location and sunspot number.

This may result in the use of a frequency which is suboptimum for propagation, but where the estimated occupancy is acceptable. It is anticipated that such occupancy models may also be useful to communication system designers, to HF ground-wave users (who may choose frequencies to avoid sky-wave interference), and to study groups who are concerned with the determination of international frequency assignments.

\subsubsection{Models}

In each case $y_{k}$ is the model index function for the kth frequency allocation $(\mathrm{k}=1,2, \ldots, 95)$, $f_{\mathrm{k}}$ is the centre frequency of allocation $\mathrm{k}(\mathrm{MHz})$, $\mathrm{E}$ is the field-strength threshold $(\mathrm{V} / \mathrm{m}), \mathrm{BW}$ is the measurement filter bandwidth $(\mathrm{kHz}), \mathrm{SSN}$ is the sunspot number, $\theta w$ defines week of year ( $2 \pi$ [week-0.5]/52-where week varies in the range 1 to 52$), \theta_{\text {long }}$ represents $(2 \pi$ longitude/360) (longitude in degrees), $\theta_{\text {lat }}$ represents $(2 \pi$ latitude/360), and A,B, .., J are estimated model coefficients. Longitudes west of the Greenwich Meridian are entered as negative values.

The minimum field-strength threshold for which the models apply has been determined from measurements of background atmospheric noise.

The sunspot number is based on the monthly average, published by the World Data Centre at the Rutherford Appleton Laboratories. It was chosen to model this parameter over two cycles to permit the extrapolation properties of the model in time to be investigated. When future values of congestion are to be estimated, it is necessary to estimate the future sunspot number.

\subsubsection{Goodness of fit}

An important consideration is goodness of fit of the model to the experimental data, where the difference between each of the measured and fitted congestion values is referred to as a residual. Statistically, goodness of fit may be defined by the residual scaled deviance $D$ which is equal to twice the difference between the maximum achievable log likelihood and that attainable under the fitted model (Maccullah and Nelder, 1989). In the analysis, the residual mean deviance $d$ is used $d=D /(R-p)$ where $R$ is the number of experimental congestion values, and $p$ is the number of independently estimated model parameters.

The scaling parameter is unity for the logistic model.

The addition of further significant parameters to a model improves the fit, and the goodness of fit is indicated by the closeness of the residual mean deviance $d$ to unity. The values of residual mean deviance for the stable-day and stable-night models are 7.8 for day and 8.2 for night, which signify very good fit to the experimental data. 


\subsubsection{Extrapolation in time and space}

To investigate the ability of the models to estimate future congestion values, a stable-day four-site model was determined independently for 355,134 congestion measurements made over the period 1990 to 1998 inclusive.

This model was then used to estimate stable-day congestion values for each of the years 1999, 2000 and 2001, using estimated sunspot number. Modelled and measured congestion values were then compared for the extrapolated periods.

The accuracy of extrapolation is very good, and is essentially equivalent for all the three years 1999, 2000 and 2001, even although the sunspot number is increasing rapidly over this period. This accuracy is in part due to the modelling only of the systematic components of the congestion data. This exercise has been repeated using a smaller data set for the model, and extrapolating over a greater time. A stable-day three-site model was derived from 219,671 measurements made over the period 1990 to 1995, which cannot include the Kiruna measurements.

This model was then used to estimate stable-day congestion values for Baldock, Linköping and Munich for each year 1996 to 2001 inclusive. The results again show that the time-extrapolation properties of the model are very good.

For extrapolation in space a stable-day three-site model was determined for congestion measurements made over the period 1990 to 2001, including data from Baldock, Linköping and Munich, but excluding data from Kiruna. The 45,280 congestion values measured at Kiruna (corresponding to four years of operation), were then estimated by the model by entering the latitude and longitude of the Kiruna site, and comparing estimated and measured congestion values. Similarly, three further three-site models were determined, excluding data from Linköping, Munich and Baldock in turn. In each case, all measured congestion values at the excluded site were estimated and compared with measured values.

Very good extrapolation in space was achieved for Kiruna and Munich, and very good interpolation for Linkoping.

The ability to extrapolate to Baldock was less good: however this may be expected as the measurements from Kiruna, Linkoping and Munich have little dependence on longitude.

\subsection{Neural network prediction of the diurnal variation of HF spectral occupancy}

The aim of this work was to investigate the possible application of artificial neural networks to predict the diurnal variation of spectral occupancy in the HF spectrum. In particular, a preliminary study for the development of a single ITU allocation model for the diurnal variation of HF spectral occupancy over Linköping (Sweden) was made. Furthermore the model specification was extended in frequency to cover the whole of the HF spectrum (all ITU allocations) and in latitude by including diurnal measurements taken at Kiruna (Sweden). The dataset of diurnal occupancy measurements used for the model development was taken over a period of six years (April 1994 to January 2000).

The model may be used to predict the occupancy that may be experienced by an HF radio system situated over Sweden, for a given set of parameters for the particular ITU allocation. The prediction of spectral occupancy in the HF spectrum can be viewed as a multivariate regression problem consisting of a nonlinear mapping from a set of input variables describing current ionospheric conditions onto a single output variable representing the likelihood of an occupied channel. Neural Networks can provide an elegant solution to this problem since with a suitable network complexity they can approximate any nonlinear mapping to an arbitrary degree of accuracy.

\subsubsection{Model development}

In the preliminary work, only measurements at Linköping for allocation 72 which is dedicated to Broadcast users (21.450 - $21.870 \mathrm{MHz})$ 

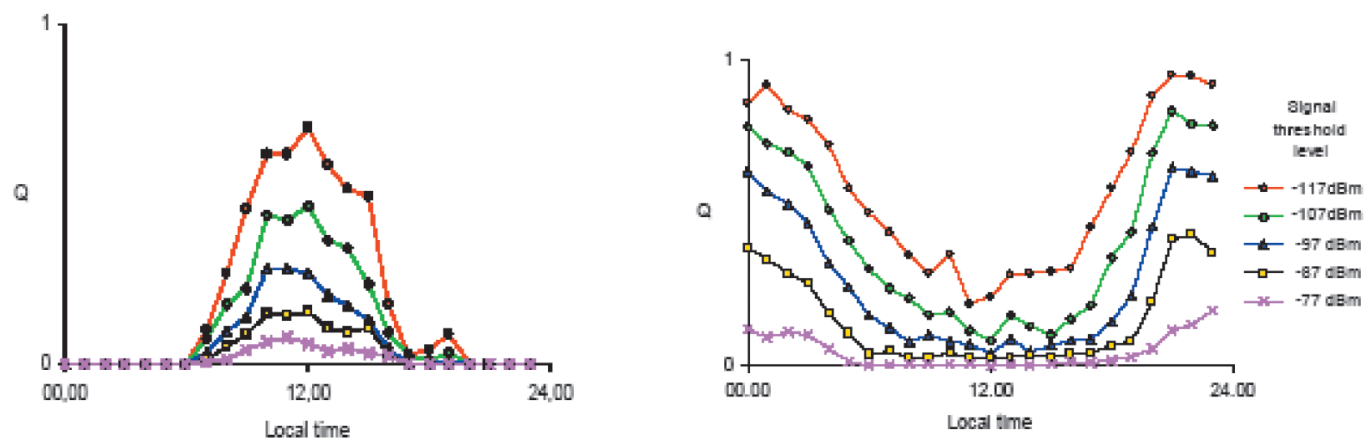

Fig. 9. Typical diurnal congestion variation in the upper and lower part of the HF spectrum.
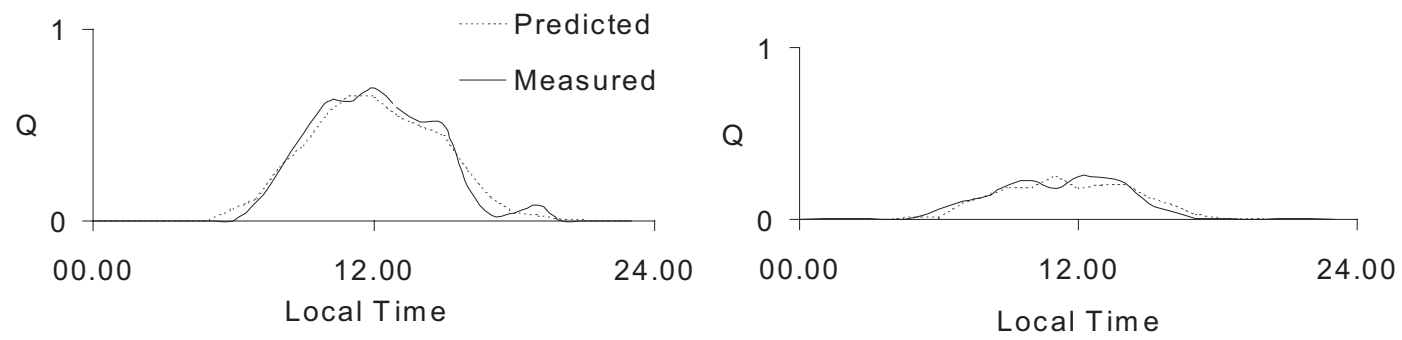

Fig. 10. Examples of diurnal measured and predicted congestion in allocation $72(21.450-21.870 \mathrm{MHz})$.

was considered in the modelling process so the congestion values per measurement taken into account were 120 . The total number of measurement sessions carried out is 197 which corresponds to a total of 23640 congestion values for the corresponding allocation. In this work the Levenberg-Marquardt backpropagation neural network algorithm was used, since it appears to be the fastest method for training moderate-sized feed-forward networks.

The variation of occupancy of the HF spectrum due to skywave signals is primarily dependant on prevailing ionospheric conditions. The input parameters for modeling congestion were selected so as to represent the known variations that give rise to the most characteristic properties of the Ionosphere as a communications channel. Solar activity has an impact on ionospheric dynamics which in turn influence the electron density of the ionosphere. Thus solar activity is proved to be highly correlated with congestion. This long-term variation was included as a separate input parameter with a running mean value of the daily sunspot number (R) which is a well-established index of solar activity. After a thorough investigation a 50-day running mean value of the daily sunspot number R50 was found to be the optimum parameter to represent the long-term variation of congestion since it achieves the minimum Root Mean Squared Error (RMSE). A short-term strong dependency between interference and the hour of the day is clearly evident by observing fig. 9. We therefore include hour numbers in the inputs to the Neural Network.

The hour number, hour, is an integer in the range $0 \leq$ hour $\leq 23$. In order to avoid unrealistic discontinuity at the midnight boundary, hour was converted into its quadrature components according to 


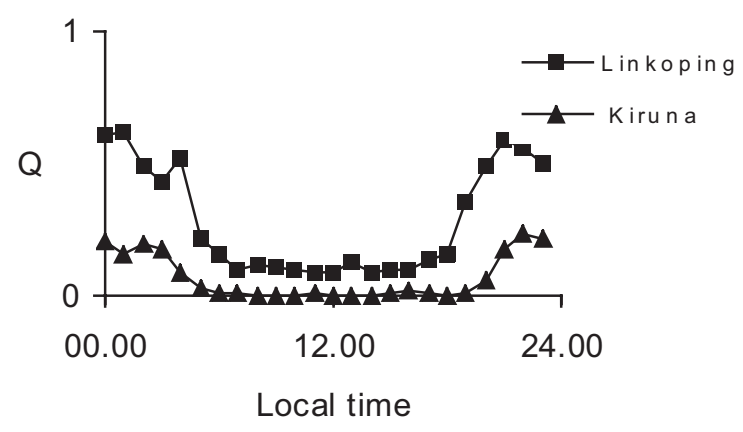

(a) 2.500-2.850 MHz

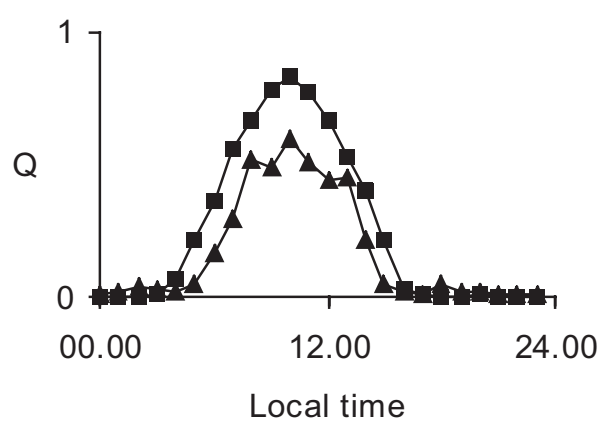

(b) 15.100-15.600 MHz

Fig. 11. Typical diurnal variation of congestion in the upper and lower HF spectrum.

$$
\operatorname{sinhour}=\sin \left(2 \pi \frac{\text { hour }}{24}\right)
$$

and

$$
\text { coshour }=\cos \left(2 \pi \frac{\text { hour }}{24}\right)
$$

A seasonal variation is described by day number daynum in the range $1 \leq$ daynum $\leq 365$. This variation is due to the response of the critical frequency of the $F 2$ layer, which acts as the principal reflector of radiowaves in the ionosphere for long-distance transmission, to the seasonal change in extreme ultraviolet (EUV) radiation from the Sun. Again to avoid unrealistic discontinuity between December 31 st and January 1st daynum was converted into its quadrature components according to

$$
\text { sinday }=\sin \left(2 \pi \frac{\text { daynum }}{365}\right)
$$

and

$$
\operatorname{cosday}=\cos \left(2 \pi \frac{\text { daynum }}{365}\right)
$$

Signal threshold level was also incorporated as an input parameter (ST) since it is obvious from fig. 9 that it has a profound effect on measured congestion. This is quite reasonable since increasing the threshold would normally correspond to fewer channels exceeding its value so this would result in a lower congestion value. The network used was a fully connected two-lay- er neural network, with 6 input, 11 hidden and 1 output neurons. Both its hidden and output neurons had logistic sigmoid activation functions. The number of hidden neurons was determined by trial and error.

This network was trained using the Levenberg-Marquardt backpropagation algorithm for a fixed number of epochs. There was no concern for overfitting since the number of free parameters of the network was very small compared to the number of examples used for its training. All inputs to the network were normalized setting the minimum value of each input to -1 and its maximum value to 1 . No normalization was needed for its outputs since they were already in the interval $(0,1)$.

The experiments were carried out using 10 random splits of the whole data set into training and test sets. More specifically, for each split 20 out of the 197 measurement sessions were randomly selected to form the test set, and the remaining 177 were used to form the training set; all 120 congestion values of each session were treated as a group and were placed either in the training or in the test set. The results reported here were obtained by averaging the results of all 10 splits. In order to determine the significance of each of the model inputs the modelling procedure was repeated five times, with each of input parameters being excluded each time. The increase in the RMSE with respect to the full parameter model was used as a quantitative 

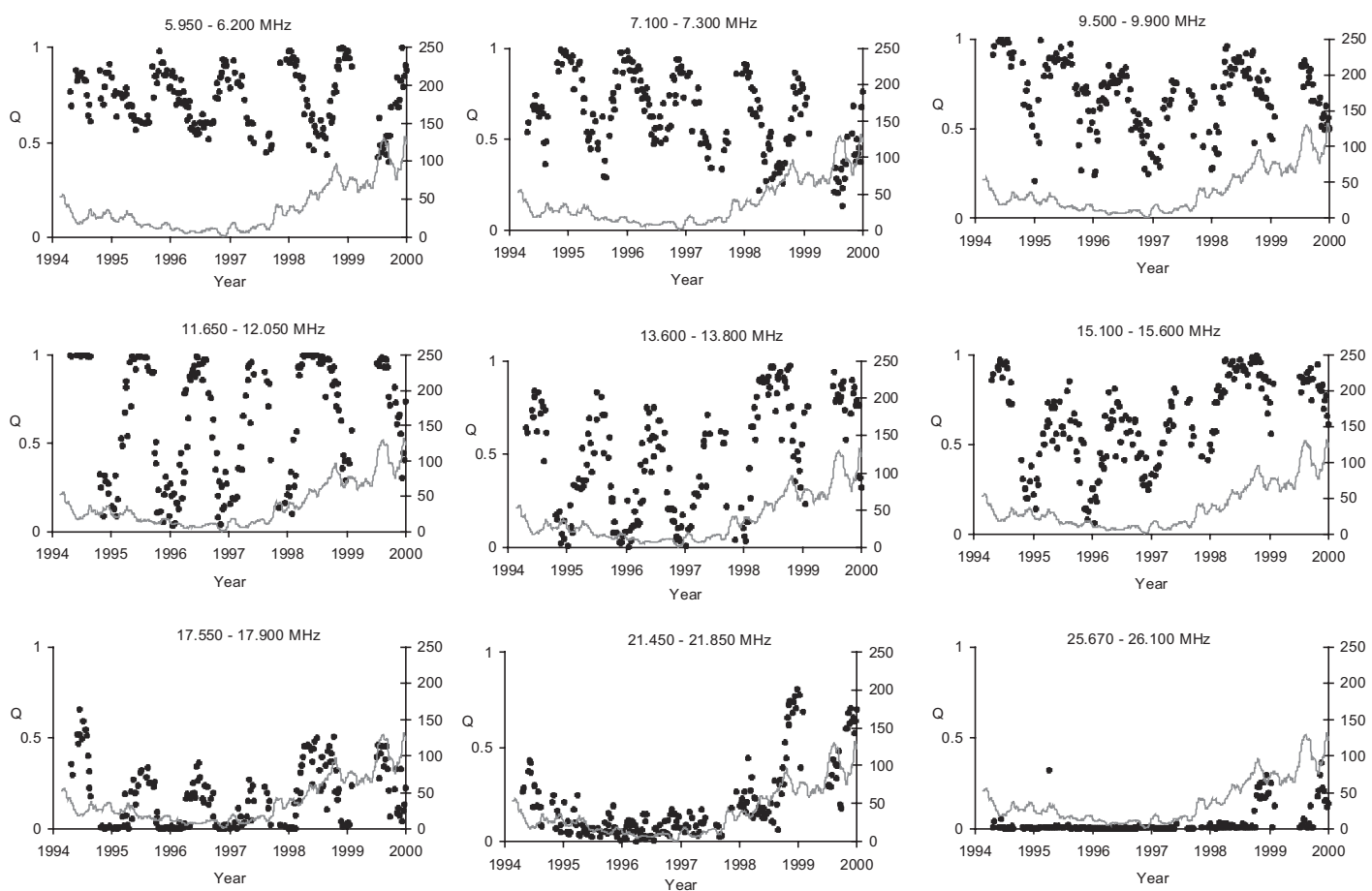

Fig. 12. Long-term and seasonal variation of congestion.

measure of the impact of each parameter to the model performance.

The RMSE and the correlation coefficient between the network predictions and the measured congestion values was 0.0284 and 0.93 respectively. Both the low RMSE value and the close to unity correlation coefficient values show that the trained neural network can successfully predict congestion in the allocation under consideration.

\subsubsection{Predicting the diurnal variation of HF spectral occupancy over Sweden}

In this work the neural network approach was applied for the prediction of the temporal variation of congestion within a frequency allocation as it was described before and extended to encapsulate the spatial and frequency dependence by including congestion measurements covering the entire HF spectrum from two measurement sites in Sweden. Examples of typical variation of measured occupancy at the two sites with timeof-day are given in fig. 11 for allocations residing in the lower and upper regions of the HF band. The variation of occupancy with time-of-day was found to differ significantly across the HF band. Allocations in the lower portion of the HF band exhibit different characteristics to those residing in the upper portion of the band. An example of typical occupancy encountered in the lower portion of the HF band is given in fig. 11a, from which significant diurnal variation of congestion can be observed, peaking during night-time. Conversely, in the upper portion of the HF band a complete reversal of diurnal variation is observed as shown in fig. $11 \mathrm{~b}$, which again shows significant diurnal variation, but in this case occupancy is highest during day-time. 
During years of low solar activity, the lower frequencies tend to be utilized more, while during years of high solar activity the higher frequencies are also used. This is demonstrated in fig. 12 where congestion measured at Linköping is plotted (with dots) for Broadcast allocations distributed across the HF spectrum together with the 50-day running mean of the daily sunspot number (depicted as a continuous line in the background).

This figure shows that congestion is significantly higher for high sunspot number periods in allocations $72(21.450-21.850 \mathrm{MHz})$ and 86 (25.670 - $26.100 \mathrm{MHz}$ ).

The same figure also demonstrates the seasonal variation of congestion in each allocation. This is attributed to the seasonal variation of the critical frequency of the F2 layer, which acts as the principal reflector of radiowaves in the ionosphere for long-distance transmission, due to its response to seasonal change in extreme ultraviolet (EUV) radiation from the Sun. Both solar cycle and seasonal variation are manifested differently in each allocation underlying the different occupancy characteristics at each frequency. In allocation $22(5.950-6.200 \mathrm{MHz})$ the increasing sunspot number appears to ex- hibit an increasing amplitude of seasonal variation whereas in allocation 27 (7.100 - 7.300 $\mathrm{MHz}$ ) it seems to have a decreasing effect on the average congestion level. In allocations 42 (11.650 - $12.050 \mathrm{MHz}), 48$ (13.600 - 13.800 $\mathrm{MHz})$ and $53(15.100-15.600 \mathrm{MHz})$ residing in the middle of the HF spectrum the solar cycle effect appears as an increasing average and a decreasing amplitude in the seasonal congestion variation.

\subsubsection{Model development}

A Neural Network was trained to predict the congestion in each allocation based on the input parameters. The neural networks used were fully connected two-layer networks with 7 input (including a binary parameter to indicate the site of the measurement) and 1 output neurons. Both their hidden and output neurons had logistic sigmoid activation functions. A different number of hidden neurons was used for each allocation due to their difference in complexity and occupancy characteristics. The optimum number of hidden neurons was determined by forward selection. Each network was trained
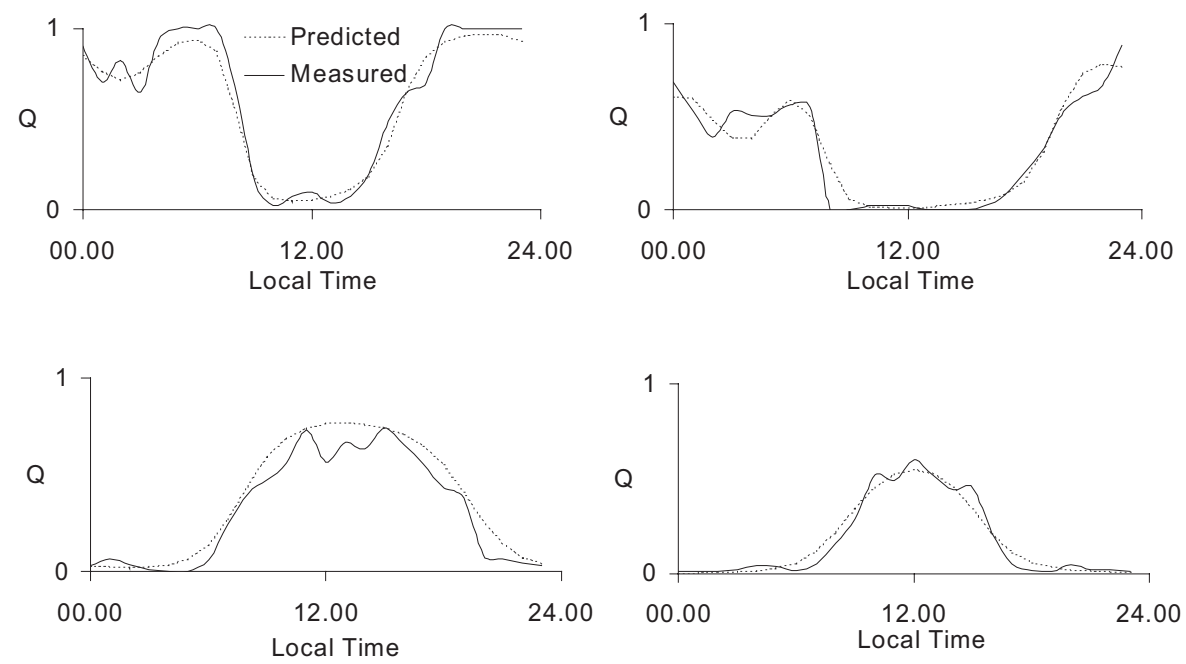

Fig. 13. Examples of diurnal measured and predicted congestion over Sweden. 

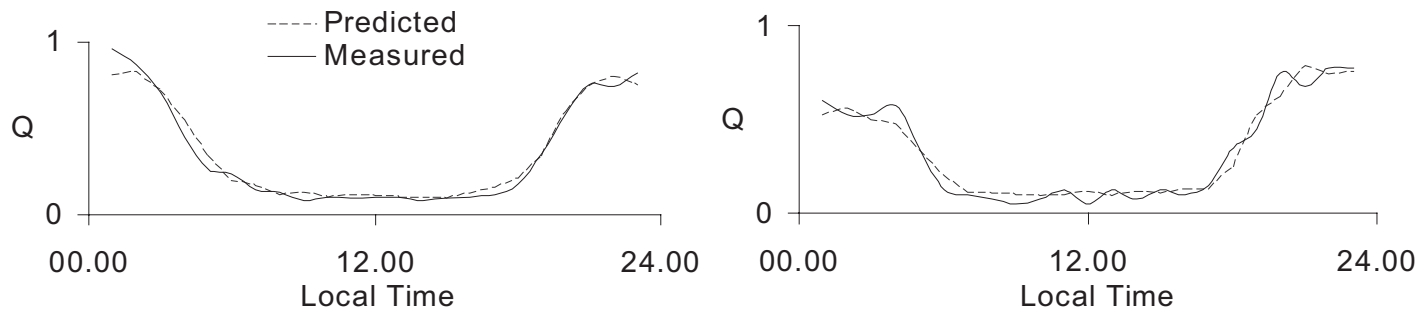

Fig. 14. Examples of diurnal measured and predicted congestion over Sweden.

using the Levenberg-Marquardt backpropagation algorithm for a fixed number of epochs (100). There was no worry of overfitting since the number of free parameters of the networks was very small compared to the number of examples used for their training. All inputs to the networks were normalized setting the mean value of each input to 0 and its standard deviation to 1 . The experiments were carried out using a 10 -fold cross-validation procedure.

The 268 measurement sessions were split into 10 parts of almost equal size and the predictions for each part were obtained using a neural network trained on the examples of the other 9 parts. Both the low RMSE value and the close to unity correlation coefficient values for most allocations, demonstrated that the trained neural networks can successfully predict congestion. This is also demonstrated by examples of measured and predicted congestion values (fig. 13).

\subsection{Using neural networks for short-term forecasting the likelihood of interference to groundwave users in the HF spectrum}

In this study a Neural Network approach was proposed for the short-term forecasting of the likelihood of interference experienced by HF groundwave communication systems. In particular this section describes the development of neural network models to indicate the degree of spectral congestion in frequency allocations in the lowest part of the HF spectrum (1.6 to $4 \mathrm{MHz}) 1$ hour in advance, as a function of the present congestion level, time of day, season, and field strength threshold. This study was a continuation of previous work (Haralambous et al. 2007), where Neural Networks were used for the prediction of interference when there is no means of measuring it. In this paper the possibility for short-term forecasting of the same parameter is explored in the context of near real time channel evaluation (RTCE), which implies recent measurements of the target model parameter incorporated as inputs to the neural network. This approach is applicable in the case of a system which is capable of measuring interference and can use that information to obtain a short-term forecast of the measured parameter 1 hour in advance.

\subsubsection{Model parameters}

The parameters used to describe the diurnal and seasonal variation were again sinusoidal components identical to those described in Section 2.5.1. Regarding the effect of solar activity a further investigation revealed that the congestion variation due to the long-term variation of solar activity is to a large extent described by the current value of congestion $Q(\mathrm{~h})$ (where $\mathrm{h}$ is the current hour) which was included as an additional model input. Of course this implies that the system should be able to measure the current congestion value in order to forecast the next congestion value $Q(\mathrm{~h}+1) 1$ hour in advance. This characteristic poses a significant requirement on the system in question but on the other hand allows for the model to operate without external data to express the solar activity conditions which would necessitate a provision of an updated solar index figure. Signal threshold level was 
also incorporated as an input parameter since it has a profound effect on measured congestion.

\subsubsection{Results}

In this study each neural network was trained to forecast the congestion $Q(\mathrm{~h}+1)$ of a given allocation 1 hour in advance based on the sinhour (for $\mathrm{h}+1$ ), coshour, sinday, cosday, current congestion value $Q(\mathrm{~h})$ and signal threshold parameters. All networks used had exactly the same structure. They were fully connected twolayer neural networks, with 6 input, 5 hidden and 1 output neurons. Both their hidden and output neurons had logistic sigmoid activation functions. The number of hidden neurons was determined by trial and error. More specifically, networks with 3 to 15 hidden neurons were tried and our choice was based on the fact that in most cases there was no significant reduction in the RMSE when increasing their number to more than 5 . These networks were trained using the Levenberg-Marquardt backpropagation algorithm for a fixed number of epochs (100).

\subsection{Conclusions}

Models of HF occupancy over northern Europe have been studied for a complete sunspot cycle. Very good accuracy of fit is achieved to the very large experimental data set. The accuracy with which the models may be extrapolated in time and in space has also been shown to be very good. In particular, the extrapolation using the models in both time and in space to estimate congestion at Kiruna is considered excellent, especially given the high latitude of the site and the rapidly changing sunspot number over the period of extrapolation. Neural network models have been developed to describe the diurnal variation of occupancy within frequency allocations of the HF spectrum. The models were developed from extensive diurnal occupancy measurements taken over a period of six years. The remarkable agreement between the neural network predicted values and observed congestion data signifies the ability of the resulting models to provide a quantitative description of the diurnal, seasonal and long-term trend in the variability of congestion. The proposed networks require the day number, hour, 50-day running mean of the daily sunspot number and signal threshold as inputs, and predict the corresponding congestion value, which indicates the likelihood of interference under the given conditions.

Finally measurements over the HF band during the 29 March 2006 total solar eclipse in Antalya revealed the variation of atmospheric noise under the eclipse conditions.

\section{Acknowledgements}

The authors are grateful to Prof G.F. Gott, Prof P.J. Laycock and P.R. Green of the University of Manchester for providing relevant background experience, and to M. Bröms, S. Boberg and Bengt Lundborg (FOI Swedish Defence Research Agency, Linköping, Sweden) for supplying the measurement data for the development of the neural networks.

\section{REFERENCES}

Azevedo, J.A. (2001): Application of Signal Processing to the Analysis and Synthesis of Antenna Arrays, Ph.D. Thesis, (Faro, Portugal).

AzEvEDO, J.A. (2007): Antenna pattern control of planar arrays for long-range communications, IRI/COST 296 Workshop on Ionosphere Modeling, Forcing and Telecommunications, (Prague, Czech Republic).

CAsimiro, A.M. (1990): The Basic Relation of Radiation, Ph.D. Theis, (Porto, Portugal).

CAsimiro, A.M. and J.A. Azevedo (2005): A Unification Procedure to the Analysis and Synthesis of Antenna Arrays, Journal of Electromagnetic Waves and Applications, 19 (14), 1881-1896.

CoHEN, H. (1992): Mathematics for Scientists \& Engineers, (Prentice-Hall International Editions).

Economou, L.V., H. Haralambous, C.A. Pantjiaros, P.R. Green, G.F. GotT, P.J. LAYCOCK, M. Broms and S. Boberg (2005): Models of HF Ocucupancy over a Sunspot Cycle, IEE Proc-Commun, 152 (6), 980-988.

ElLIOT, R.S. (1981): Antenna theory and design, (PrenticeHall).

GotT, G.F., S.K. Chan, C.A. PANTJiaros and P.J. LAYCOCK (1997): High frequency spectral occupancy at the solstices, IEE Proc.-Commun, 144 (1), 24-32.

Grilo, F.V. and A.M. CASIMIRo (1991): The Basic Relation Between Point Sources and the Produced Fields, Presented at the Progress In Electromagnetics Research Symposium, (Cambridge, USA).

Hansen, R.C. (1998): Phased Array Antennas, (John Wiley \& Sons, Inc.) 
Haralambous, H. and H. Papadopoulos (2007): Neural Network Prediction of HF Spectral Occupancy, HF07, Nordic Shortwave Conferencee, (Sweden).

Haralambous, H. and H. Papadopoulos (2008): Shortterm Forecasting of the Likelihood of Interference to Groundwave Users in the Lowest Part of the HF Spectrum $I S^{\prime} 08$.

Haralambous, H., H. Papadopoulos and L.V. Economou (2007): Using Neural Networks for Predicting the Likelihood of Interference to Groundwave Users in the HF Spectrum EANN07.

Haralambous, H., H. Papadopoulos and L.V. ECONOMOU (2008): Predicting the diurnal variation of HF spectral occupancy over Sweden IES2008.

Maccullah, P. and J.A. Nelder (1989): Generalised linear models, (Chapman and Hall).

Tulunay, E., E.M. Warkington, Y. Tulunay, Y. BAHADIRLAR, A.S. TURK, R. ÇAPUTÇU, T. YAPICI and E.T. SenalP (2006a): Propagation Related Measurements during Three Solar Eclipses in Turkey, IRST 2006, (1821 July 2006, London, UK).

Tulunay, E., Y. Tulunay, E.M. Warrington, Y. BAHADIRLAR, T. YAPICI, E.T. SEnAlP and A.S. TURK (2006b): Recent COST 296 Propagation Related Measurements During the 29th March 2006 Solar Total Eclipse in Antalya, Turkey, Second COST 296 Work- 